Received: 2018.11.30

Accepted: 2018.12.24

Published: 2019.04.21
Authors' Contribution: Study Design A Data Collection B Statistical Analysis C Data Interpretation D Manuscript Preparation E Literature Search F Funds Collection G

Corresponding Author: Source of support:

\section{Ferruginol Diterpenoid Selectively Inhibits Human Thyroid Cancer Growth by Inducing Mitochondrial Dependent Apoptosis, Endogenous Reactive Oxygen Species (ROS) Production, Mitochondrial Membrane Potential Loss and Suppression of Mitogen-Activated Protein Kinase (MAPK) and PI3K/AKT Signaling Pathways}

\author{
BCE Guoqing Luo \\ $B C D F$ Jingjing Zhou \\ BEF Guanjie Li \\ BDE Ningdong $\mathrm{Hu}$ \\ BCDF Xu Xia \\ ACDG Haibo Zhou
}

Background: Thyroid cancer causes considerable mortality and morbidity across the globe. Owing to the unavailability of biomarkers and the adverse effects of existing drugs, there is an urgent need to develop efficient chemotherapy for the treatment of thyroid cancers. Plants have served as exceptional source of drugs for the treatment of lethal diseases. The purpose of this study was to evaluate the anticancer effects of ferruginol against thyroid cancer cells.

Material/Methods: We monitored the cell proliferation rate using 3-(4,5-dimethylthiazol-2-Yl)-2,5-diphenyltetrazolium bromide (MTT) assay. Apoptosis was detected using 4',6-diamidino-2-phenylindole (DAPI), acridine orange/ethidium bromide (AO/EB), and annexin V/propidium iodide (PI) staining. Reactive oxygen species (ROS) and mitochondrial membrane potential (MMP) levels were examined by fluorescence microscopy. Protein expressed was examined by western blotting.

Results: We found that ferruginol exerted potent antiproliferative action against thyroid cancer cells, and an $I C_{50}$ of $12 \mu \mathrm{M}$ was observed for ferruginol against the MDA-T32 cell line. The toxic effects of ferruginol were less pronounced against normal cells. The anticancer effects of ferruginol were likely due to the induction of apoptosis which was also associated with upregulation of Bax and downregulation of $\mathrm{Bcl}-2$. Ferruginol also caused ROS mediated alterations in the MMP of MDA-T32 cells. In MDA-T32 cells, ferruginol might also block the MAPK and PI3K/AKT signaling pathway, which is believed to be an important therapeutic target of anticancer drugs.

Conclusions: In conclusion, in view of the results of this study, it might be suggested that ferruginol might serve as an essential lead molecule for the treatment of thyroid cancer provided further in-depth studies especially studying ferruginol toxicological as well as in vivo studies are needed.

MeSH Keywords: $\quad$ Apoptosis Regulatory Proteins • Cell Proliferation • Parathyroid Neoplasms • Reactive Oxygen Species

Full-text PDF: https://www.medscimonit.com/abstract/index/idArt/914348

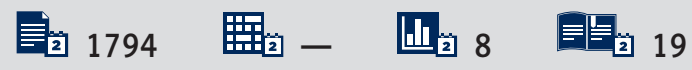




\section{Background}

Plants face exceptionally harsh and stressful environmental conditions and to save itself from these extremes; plants have developed an amazing capacity to biosynthesize a wide array of chemical entities. These chemical entities have diverse structures and have been shown to have the potential to alleviate human ailments [1]. Diterpenoids include a large group of plant secondary metabolites [2]. These diterpenoids have been shown to have immense pharmacological potential [2]. They have been shown to suppress the growth of cancer cells; for instance, diterpenoid constituents of Salvia plants have been observed to cause the apoptotic cell death of cancer cells through different modes of action [3]. Moreover, the diterpenoids extracted from the plants of Picea glehni have also been shown to curb the growth of cancer cells [4]. Ferruginol is an important diterpenoid that might prove to have immense pharmacological potential [5]. It has been shown to inhibit the growth of cancer cells such as prostate cancer and nonsmall lung cancer $[5,6]$. However, very little research efforts have been made to examine the anticancer effects of ferruginol against thyroid cancer. In this study, we for the first time evaluated the anticancer effects of ferruginol against MDA-T32 thyroid cancer cells. Thyroid cancer is one of the less conmen but lethal type of cancer. The incidence of thyroid cancer is increasing and is the cause of significant mortality throughout the world [7]. The late diagnosis and the metastasis of thyroid cancer makes it very difficult to manage [8]. Generally, surgical innervations and chemotherapy are used for the management of thyroid cancer [9]. However, the currently available drugs impair the quality of life of the patients due their adverse effects [10]. In the present study, the anticancer effects of ferruginol were examined for the first time against MDA-T32 thyroid cancer cells and normal human thyrocyte cells. We found that ferruginol caused a concentration-dependent decline in the viability of the MDA-T32 cancer cells with a minimal cytotoxic effect on normal MDA-T32 cells, suggesting selective anticancer effects of ferruginol. Studies have reported that the MAPK and PI3K/AKT pathways could prove beneficial targets for anticancer drugs [11,12]. Thus, we also investigated the effect of ferruginol on MAPK and PI3K/AKT pathways in thyroid cancer cells.

\section{Material and Methods}

\section{Cell culture conditions}

The thyroid cancer cell line MDA-T32 and normal human thyrocyte were obtained from the Cancer Research Institute of Beijing (Beijing, China) and maintained in Dulbecco's modified Eagle's medium (Invitrogen Life Technologies, MA, USA) supplemented with $10 \%$ fetal bovine serum (Invitrogen Life Technologies,
MA, USA), $100 \mu \mathrm{g} / \mathrm{mL}$ streptomycin, and $100 \mathrm{U} / \mathrm{mL}$ penicillin $\mathrm{G}$ (HiMedia, PA, USA) in an incubator at $37^{\circ} \mathrm{C}$ with $5 \% \mathrm{CO}_{2}$.

\section{Cell viability assay}

The viability of the cancer cells was measured using 3-(4,5-dimethylthiazol-2-Yl)-2,5-diphenyltetrazolium bromide (MTT) assay. In brief, as the confluence of the MDA-T32 and normal human thyrocyte cells reached around $70 \%$ confluence, they were seeded in 96-well plates and treated with 0 to $160 \mu \mathrm{M}$ of ferruginol ( $98 \%$ by HPLC, purchased from Sigma-Aldrich). After an incubation of 24 hours, the cells were incubated with MTT for 4 hours. After this, the media was removed, and the colored formazan product was solubilized by $200 \mu \mathrm{L}$ of dimethyl sulfoxide. The viability of the MDA-T32 cells and the normal human thyrocyte cells was then determined by taking absorbance at $570 \mathrm{~nm}$ by using a spectrophotometer (BD Biosciences, San Jose, CA, USA).

\section{Acridine orange/ethidium bromide (AO/EB), 4',6-diamidino-2-phenylindole (DAPI), and annexin $\mathrm{V} /$ propidium iodide $(\mathrm{PI})$ staining}

The MDA-T32 cells were grown in 6-well plates $\left(0.6 \times 10^{6}\right.$ cells/well) and subjected to incubation for 12 hours. The MDA-T32 cells were subjected to ferruginol $(0,6,12$, and $24 \mu \mathrm{M})$ treatment for 24 hours at $37^{\circ} \mathrm{C}$. As the cells sloughed off, $10 \mu \mathrm{L}$ of cell culture was place on a glass slides and subjected to staining solution of acridine orange/ethidium bromide (AO/EB). The slides were then covered with a covers lip and examined with a fluorescent microscope. Then 4',6-diamidino-2-phenylindole (DAPI) and annexin V/propidium iodide (PI) staining was performed as described previously [13].

\section{Determination of reactive oxygen species (ROS) and mitochondrial membrane potential (MMP)}

The reactive oxygen species (ROS) and mitochondrial membrane potential (MMP) levels were estimated by culturing MDA-T32 cells for 24 hours at $37^{\circ} \mathrm{C}$ and subsequently treatment with varied doses of ferruginol $(0,6,12$, and $24 \mu \mathrm{M})$ for 24 hours. Next the media was decanted, and the cells were treated with $5 \mu \mathrm{M}$ DCH-DA for estimation of ROS or rhodamine 123 (Rh123) for estimation of MMP by examining the cells under laser scanning confocal Microscope (BD Biosciences, San Jose, CA, USA).

\section{Western blotting}

The MDA-T32 cells were harvested and subjected to washing with ice-cold PBS (phosphate buffered saline). The pellet was then suspended in a lysis buffer at $4^{\circ} \mathrm{C}$ and then shifted to $95^{\circ} \mathrm{C}$. Thereafter, the protein content of each cell extract was checked by Bradford assay. About $40 \mu \mathrm{g}$ of protein was loaded 
from each sample and separated by sodium dodecyl sulfate polyacrylamide gel electrophoresis (SDS-PAGE) before being shifted to polyvinylidene fluoride membrane. The membranes were then subjected to treatment with TBS (Tris-Hcl buffered saline) and then exposed to primary antibodies at $4^{\circ} \mathrm{C}$. Thereafter, the cells were treated with appropriate secondary antibodies and the proteins of interest were visualized by enhanced chemiluminescence reagent.

\section{Statistical analysis}

The experiments were performed in triplicate and data are shown as mean \pm standard deviation (SD). Statistical analysis was done using Students $t$-test with GraphPad Prism 7 software. Values of $P<0.05$ were taken as statistically significant difference.

\section{Results}

\section{Ferruginol inhibited the proliferation of MDA-T32thyroid cancer cells}

The effects of ferruginol on the viability of MDA-T32 and normal human thyrocyte cells were studied by MTT assay at concentrations ranging from 0 to $160 \mu \mathrm{M}$. The $\mathrm{IC}_{50}$ were calculated by absorbance at $570 \mathrm{~nm}$ using a spectrophotometer. It was observed that ferruginol suppressed the growth of the MDA-T32 cells in a dose dependent manner (Figure 1). The IC $\mathrm{I}_{50}$ of ferruginol against the MDA-T32cells was found to $12 \mu \mathrm{M}$. Nonetheless, the effects of ferruginol on the viability of the normal human thyrocyte cells were less pronounced. The $I C_{50}$ of ferruginol against the normal human thyrocyte cells was $92 \mu \mathrm{M}$ (Figure 1).

\section{Ferruginol induced apoptotic cell death of MDA-T32 cells}

To decipher the mechanism behind the antiproliferative effects of ferruginol on MDA-T32 cells, the ferruginol treated MDA-T32 cells were subjected to staining with AO/EB and DAPI. The AO/EB staining showed that the orange colored cells increased in number as the concentration of ferruginol was increased (Figure 2). Similarly, the DAPI staining indicated that the cells with white colored nuclei increased indicative of apoptosis (Figure 3). The annexin V/PI staining showed that the percentage of apoptotic cells in the untreated cell samples was $5.6 \%$ and as the concentration of ferruginol was increased to $24 \mu \mathrm{M}$, the apoptotic cell percentage increased to around $61 \%$, indicative of the dose-dependent apoptotic effects of ferruginol (Figure 4).

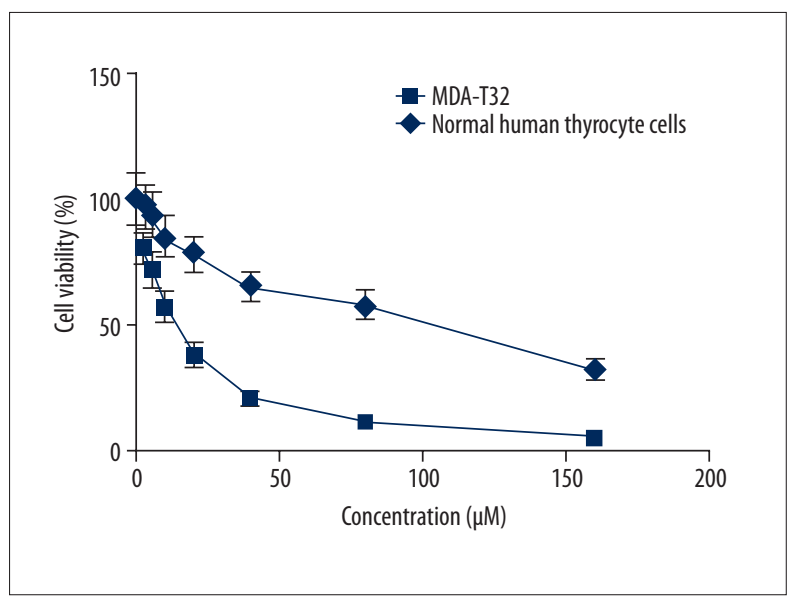

Figure 1. MTT assay showing the effect of ferruginol on the viability of the thyroid cancer MDA-T32 and normal human thyrocyte cells. The figure depicts that ferruginol inhibits the growth of thyroid cancer cells dose dependently. The experiments were performed in triplicate and shown as mean \pm standard deviation ( $\left.{ }^{*} P<0.05\right)$. MTT - 3-(4,5-dimethylthiazol-2-Yl)-2,5diphenyltetrazolium.

\section{Effects of ferruginol Bax and Bcl-2 expression in MDA-T32 cells}

The effects of ferruginol on the Bax and $\mathrm{Bcl}-2$ expression of MDA-T32 cells were examined as Bax and Bcl-2 are considered important biomarker proteins of apoptosis. The results of the western blot analysis showed that Bax expression increased dose dependently and that $\mathrm{Bcl}-2$ expression decreased, which was indicative of apoptosis (Figure 5).

\section{Ferruginol caused accretion of ROS in MDA-T32 cells}

The effects of ferruginol at different concentrations $(0,6$, 12 , and $24 \mu \mathrm{M}$ ) on the MDA-T32 cells were examined by fluorescence microscopy. The results showed that ferruginol caused significant increases in the ROS levels of MDA-T32 cells (Figure 6) and these effects were found to be concentration dependent.

\section{Ferruginol decreased the MMP levels of MDA-T32 cells}

Since ferruginol caused production of ROS, the effects of ferruginol at different concentrations $(0,6,12$, and $24 \mu \mathrm{M})$ on the MDA-T32 cells were examined by fluorescence microscopy. The results showed that ferruginol caused substantial decrease in the MMP levels of the MDA-T32 cells and like that of ROS, these effects were found to be concentration dependent (Figure 7). 


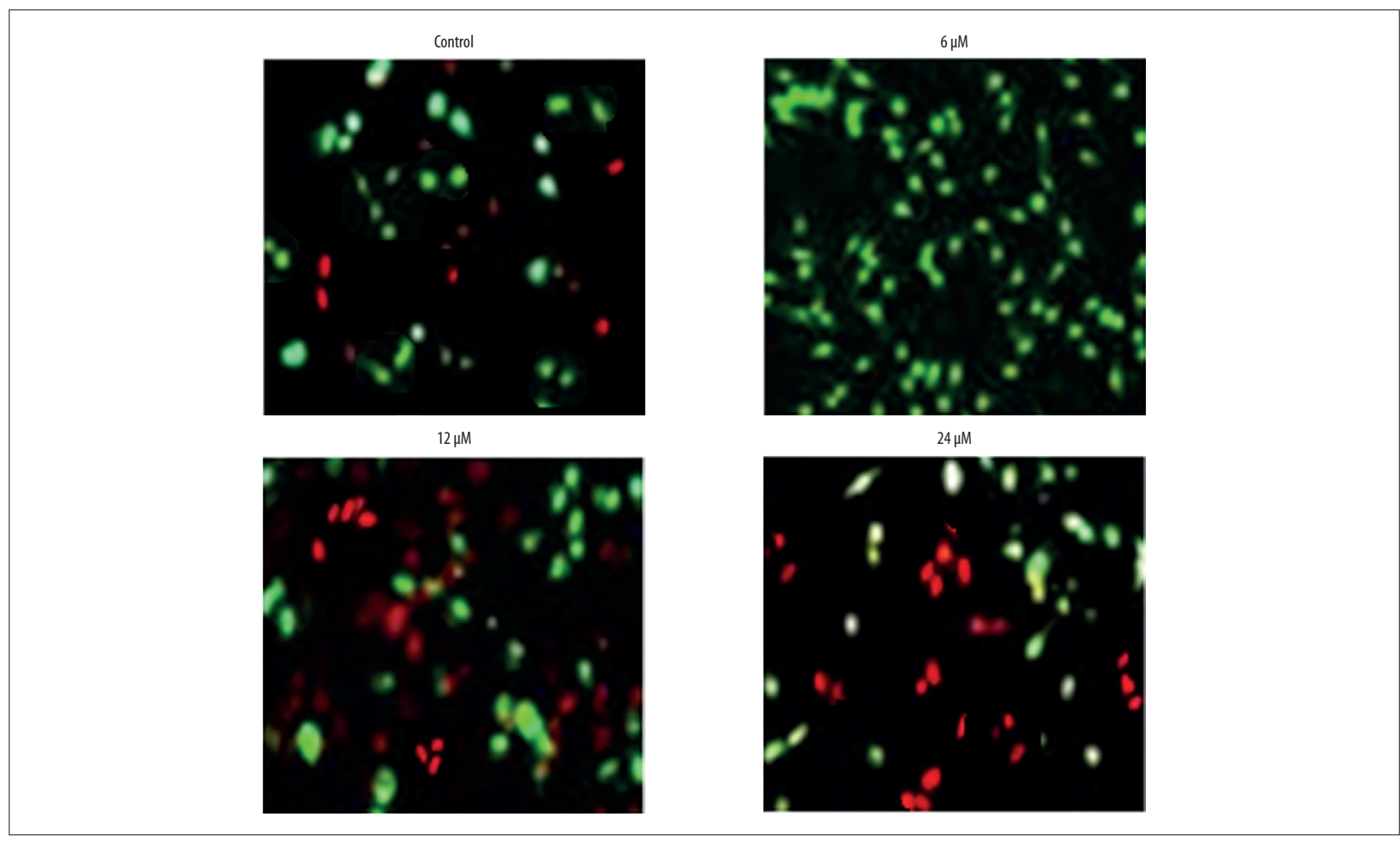

Figure 2. Induction of apoptosis in the MDA-T32 cells by ferruginol treatment as evident from the AO/EB staining. The figure depicts that ferruginol induces apoptosis in thyroid cancer cells dose dependently. The experiments were performed in triplicate. AO/EB - acridine orange/ethidium bromide.

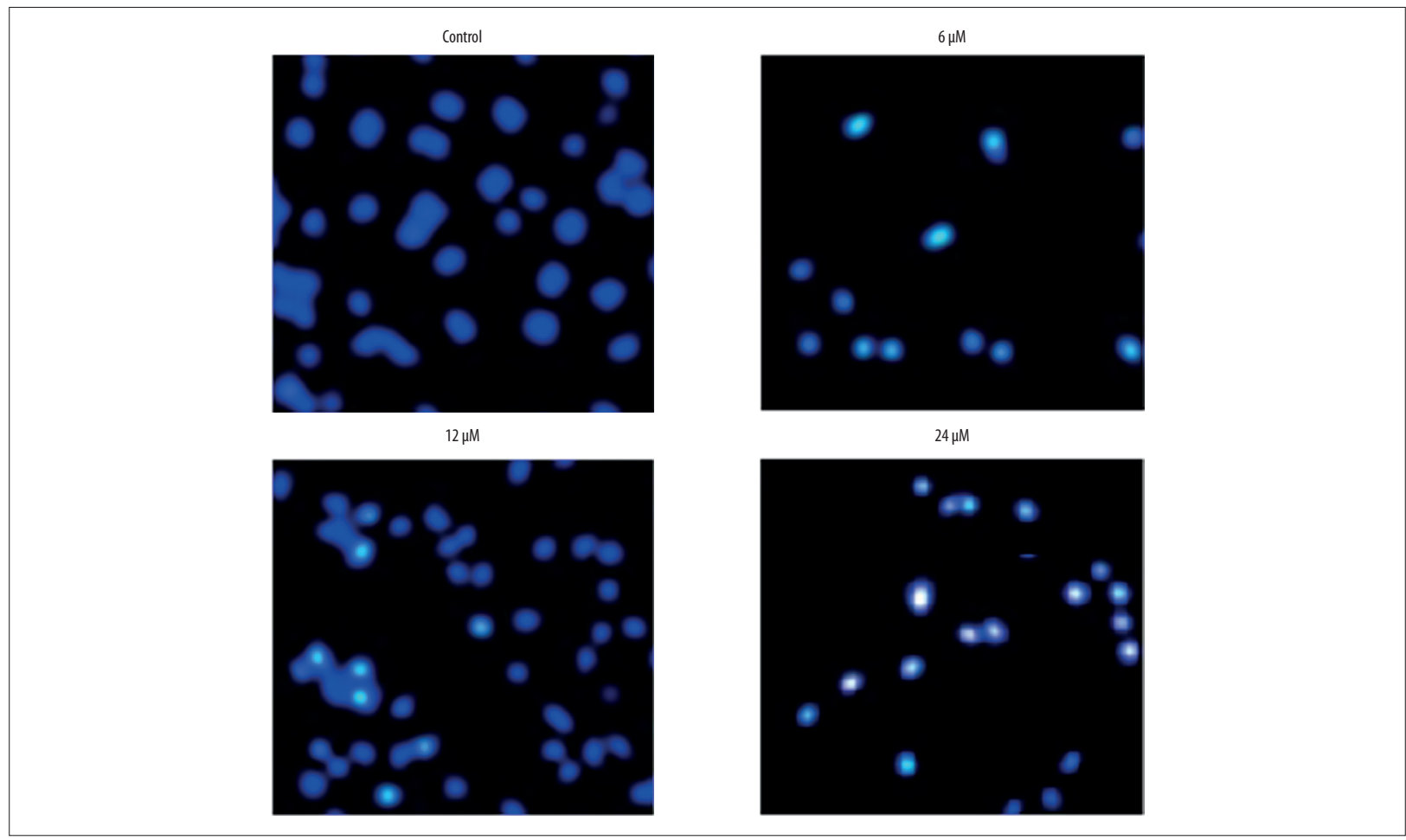

Figure 3. Induction of apoptosis in the MDA-T32 cells by ferruginol treatment as evident from the DPAl staining. The figure depicts that Ferruginol increases the DAPI positive cells concentration dependently. The experiments were performed in triplicate. DAPI - 4',6-diamidino-2-phenylindole. 


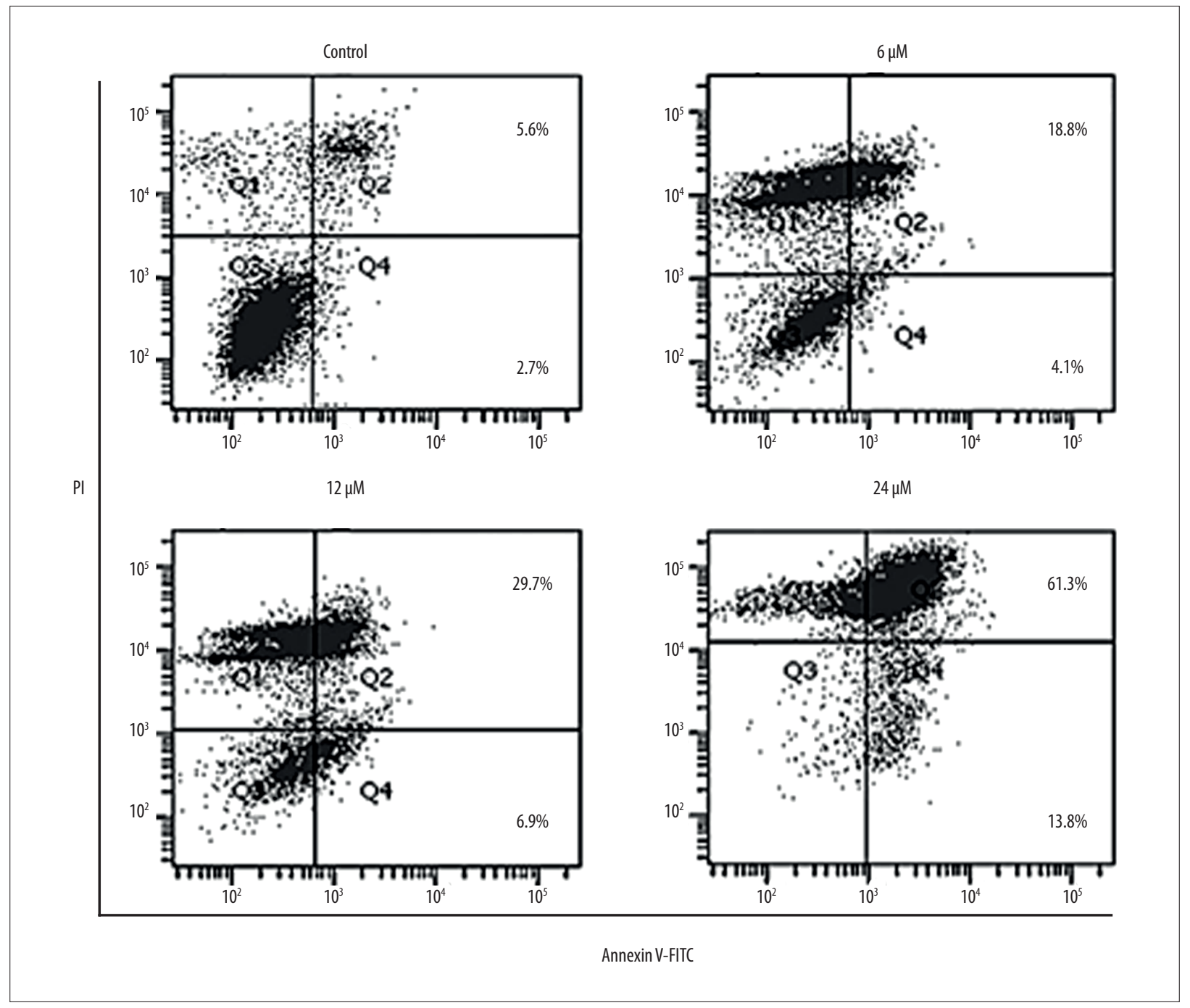

Figure 4. Annexin V/PI staining showing the percentage of apoptotic MDA-T32 cells at each phase of cell cycle. The figure depicts that ferruginol increased the percentage of the apoptotic cells in a concentration dependent manner. The experiments were performed in triplicate. $\mathrm{PI}$ - propidium iodide.

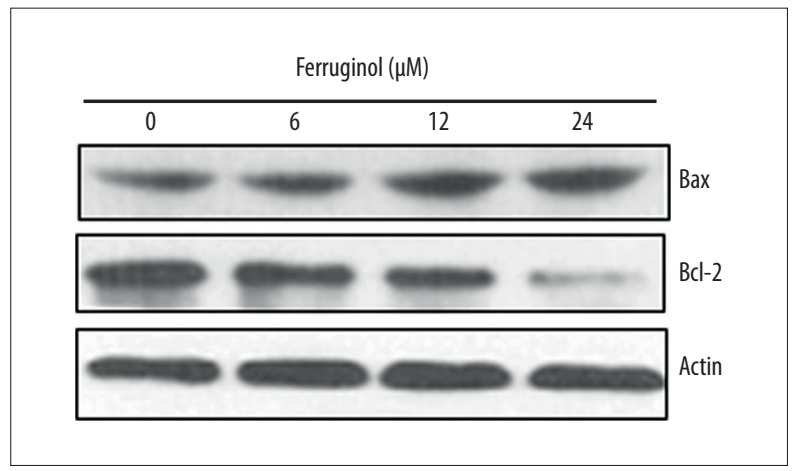

Figure 5. Effect of ferruginol on the expression apoptosis associated proteins as depicted by the western blot analysis. The experiments were performed in triplicate.

\section{Ferruginol inhibited the MAPK and PI3K/AKT signaling pathway of MDA-T32 cells}

The MAPK and PI3K/AKT/mTOR signaling pathways are considered an important therapeutic targets for the treatment of cancer. Hence, the effect of ferruginol on these signaling pathways was also examined. The results showed that ferruginol caused a decrease in the expression of p38 MAPK (Figure 8A). Moreover, ferruginol also caused a considerable decrease in the expression of $\mathrm{p}$-PI3K and $\mathrm{p}-\mathrm{AKT}$ in a dose dependently manner while no effect was found on total PI3K and AKT protein expression (Figure 8B). 


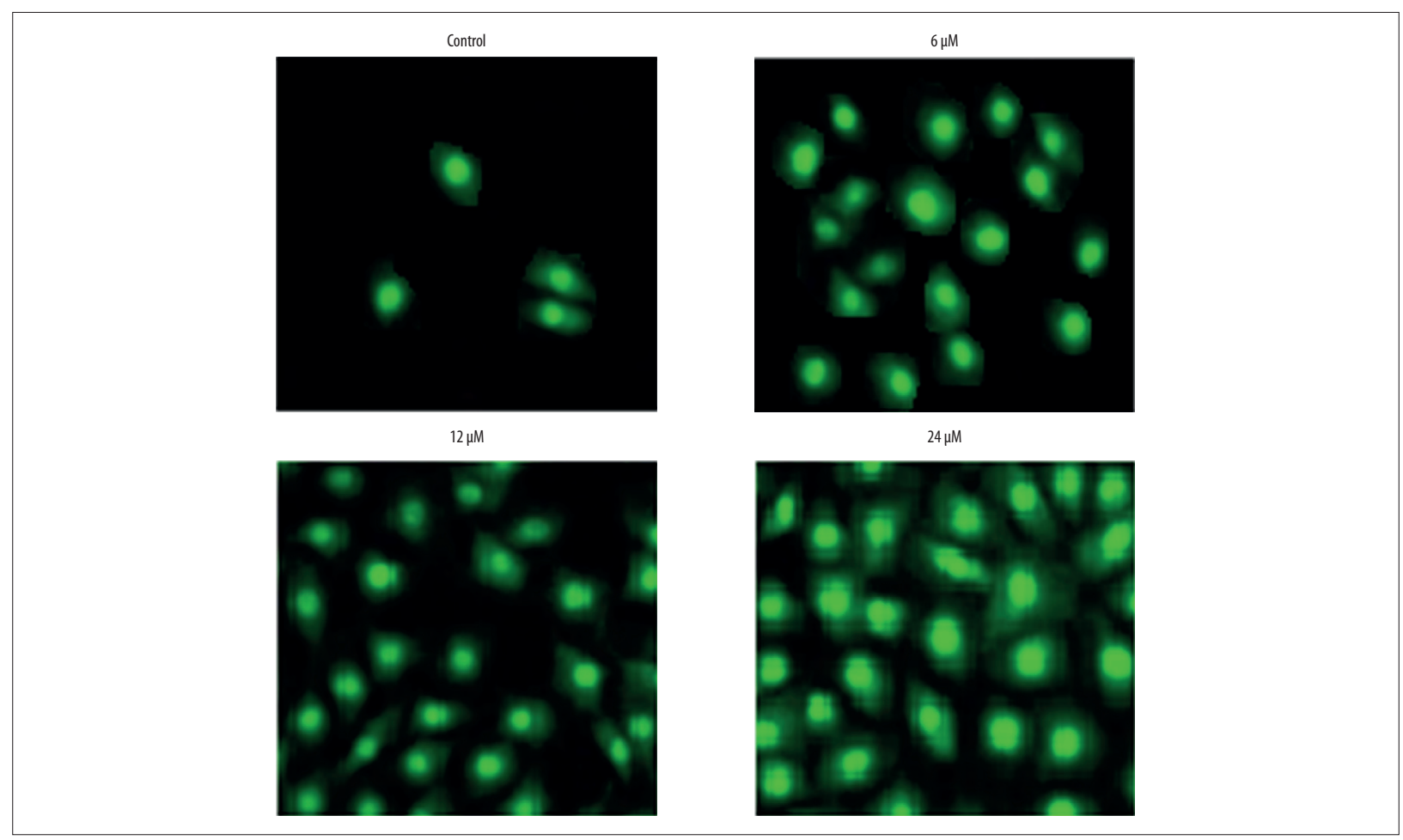

Figure 6. Fluorescence microscopy of ferruginol treated MDA-T32 cells showing increase in ROS and decrease in MMP levels of the MDA-T32 cells. The experiments were performed in triplicates. ROS - reactive oxygen species; MMP - mitochondrial membrane potential.

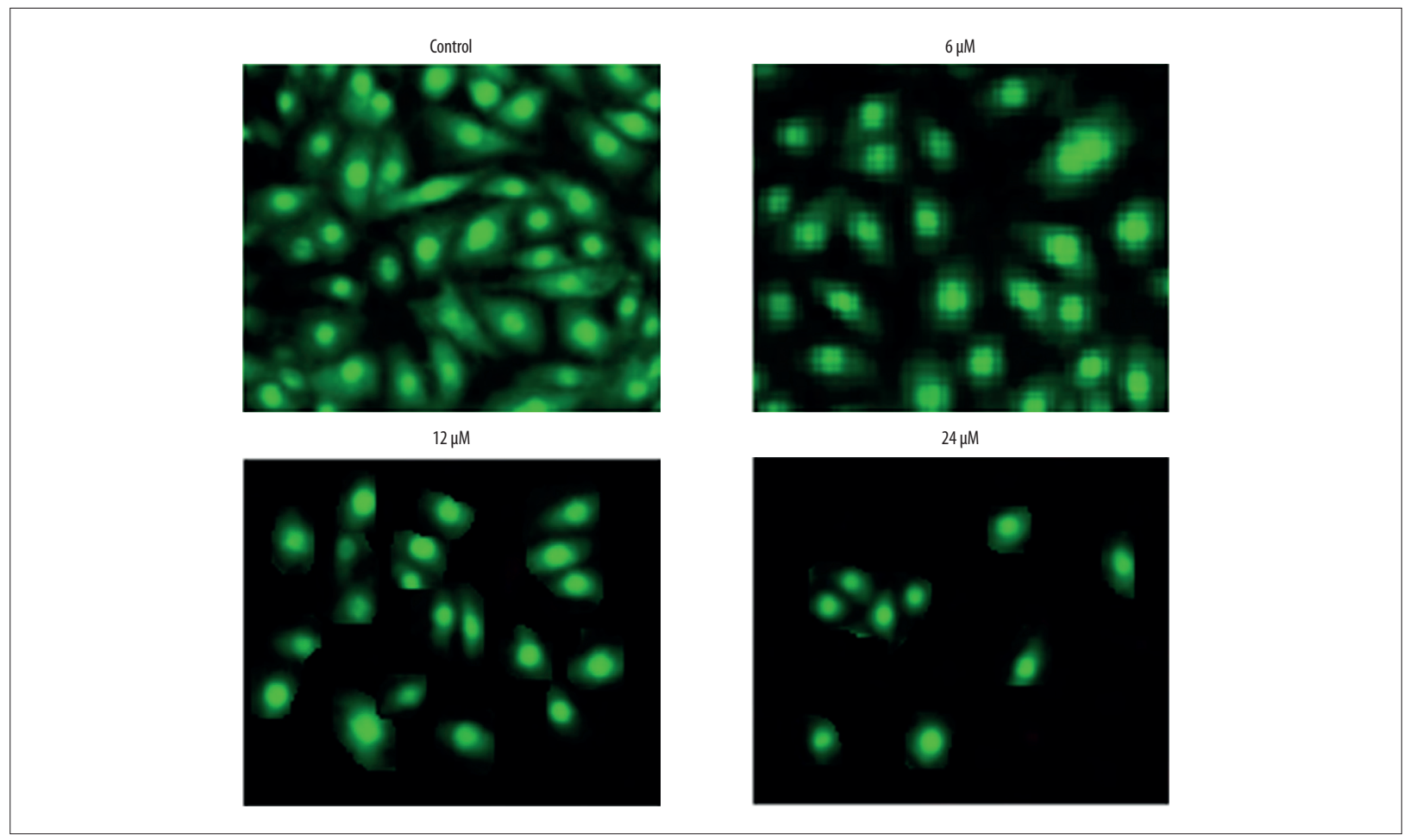

Figure 7. Fluorescence microscopy of ferruginol treated MDA-T32 cells showing decrease in MMP levels of the MDA-T32 cells. The experiments were performed in triplicates. MMP - mitochondrial membrane potential. 

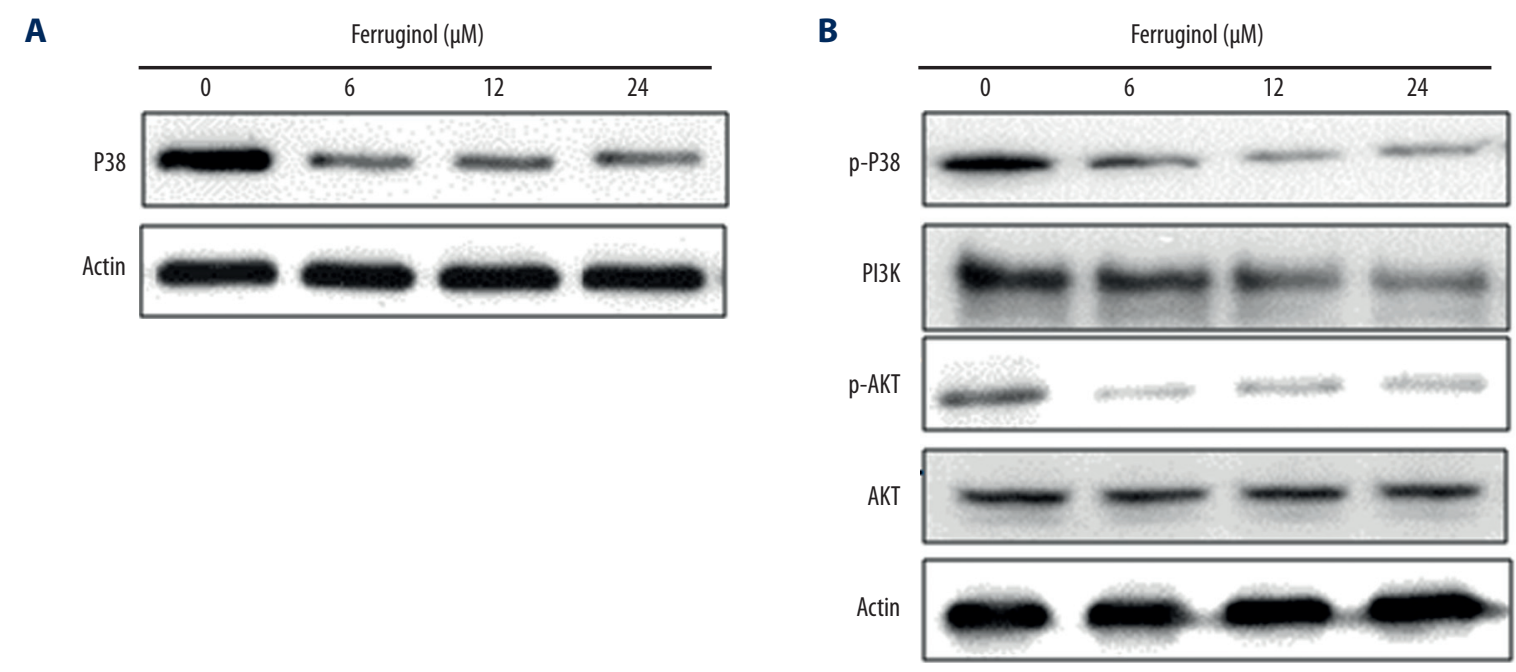

Figure 8. (A, B) Western blot analysis showing the effect ferruginol on the MAPK and PI3K/AKT/mTOR signaling pathway in HNE1 cells. The experiments were performed in triplicate.

\section{Discussion}

Thyroid cancer is one of less common and lethal types of cancer [14]. The treatment of thyroid cancer is obstructed by metastasis and the adverse effects of chemotherapeutic drugs [10]. The plant kingdom has severed as an amazing repository of molecules with potential anticancer effects which might prove beneficial in the development of chemotherapy for thyroid cancer [15]. In this study, the anticancer effects of ferruginol, a plant derived diterpenoid, against the MDA-T32 thyroid cancer and normal human thyrocyte cells were examined. It was found that ferruginol showed dose-dependent growth inhibitory effects on the MDA-T32 cells. However, ferruginol exhibited comparatively fewer toxic effects on normal human thyrocyte. cells. These results agreed with investigations that reported that ferruginol inhibited the growth of ovarian carcinoma cells and melanoma cells $[16,17]$. Compounds of plant origin have been shown to halt the growth of cancer cells through multiple mechanisms such as apoptosis, cell cycle arrest, and autophagy [18]. In this study, we observed that ferruginol decreased the viability of the MDA-T32 cells by inducing apoptotic cell death. Apoptosis of the cancer cells is essential to prevent the proliferation, metastasis, and development of chemoresistance among cancer cells [19]. The ferruginol-induced apoptosis in our study was also accompanied by upsurge of Bax and decline in Bcl-2 expression. Many plant-derived molecules have been reported to interfere with the ROS level in the cancer cells [13]. In the current study, it was found that ferruginol triggered the formation ROS in MDA-T32 cells, which was also accompanied by the disruption of MMP. The MAPK and PI3K/AKT signaling pathways are considered essential targets for anticancer drugs [11,12]. In our study, it was observed that ferruginol caused a decrease in the expression of p38 MAPK. Further, ferruginol caused a considerable decrease in the expression of $\mathrm{p}$-PI3K and $\mathrm{p}$-AKT while no effect was found on total PI3Kand AKT protein expression. Since, these results suggest potential strong anticancer effects of ferruginol; however, further evaluation of ferruginol against more cell lines and under in vivo conditions is required.

\section{Conclusions}

Ferruginol inhibited the growth of thyroid cancer cells through the induction of mitochondrial apoptosis. It also caused the production of ROS, decreased MMP levels and inhibited the MAPK and PI3K/AKT signaling pathway, indicating that ferruginol might prove beneficial for the development of chemotherapy for thyroid cancer.

\section{Conflict of interest}

None. 


\section{References:}

1. Akaberi M, Mehri S, Iranshahi M: Multiple pro-apoptotic targets of abietane diterpenoids from Salvia species. Fitoterapia, 2015; 100: 118-32

2. Kinouchi $\mathrm{Y}$, Ohtsu $\mathrm{H}$, Tokuda $\mathrm{H}$ et al: Potential antitumor-promoting diterpenoids from the stem bark of Picea glehni. J Nat Prod, 2000; 63(6): 817-20

3. Newman DJ, Cragg GM: Natural products as sources of new drugs from 1981 to 2014. J Nat Prod, 2016; 79: 629-61

4. Zhang YH, Peng HY, Xia GH et al: Anticancer effect of two diterpenoid compounds isolated from Annonaglabra Linn. Acta Pharmacol Sin 2004; 25: 937-42

5. de Jesus MB, Zambuzzi WF, de Sousa RR et al: Ferruginol suppresses survival signaling pathways in androgen-independent human prostate cancer cells. Biochimie, 2008; 90(6): 843-54

6. Ho ST, Tung YT, Kuo YH et al: Ferruginol inhibits non-small cell lung cancer growth by inducing caspase-associated apoptosis. Integr Cancer Ther, 2015; 14(1): 86-97

7. Davies L, Welch HG: Increasing incidence of thyroid cancer in the United States, 1973-2002. JAMA, 2006; 295(18): 2164-67

8. Sipos JA, Mazzaferri EL: Thyroid cancer epidemiology and prognostic variables. Clin Oncol, 2010; 22(6): 395-404

9. De Crevoisier R, Baudin E, Bachelot A et al: Combined treatment of anaplastic thyroid carcinoma with surgery, chemotherapy, and hyperfractionated accelerated external radiotherapy. Int J Radiat Oncol Biol Phys, 2004; 60(4): 1137-43
10. Coates A, Abraham S, Kaye SB et al: On the receiving end - patient perception of the side-effects of cancer chemotherapy. Eur J Cancer Clin Oncol, 1983; 19(2): 203-8

11. Fresno JV, Casado E, Cejas P et al: PI3K/Akt signaling pathway and cancer. Cancer Treat Rev, 2004; 30(2): 193-204

12. Wagner EF, Nebreda AR: Signal integration by JNK and p38 MAPK pathways in cancer development. Nat Rev Cancer, 2009; 9(8): 537-49

13. Hua F, Li CH, Chen XG, Liu XP: Daidzein exerts anticancer activity towards SKOV3 human ovarian cancer cells by inducing apoptosis and cell cycle arrest, and inhibiting the Raf/MEK/ERK cascade. Int J Mol Med, 2018; 41(6): 3485-92

14. Sherma SI: Thyroid carcinoma. Lancet, 2003; 361(9356): 501-11

15. Burmistrova O, Simões MF, Rijo P et al: Antiproliferative activity of abietane diterpenoids against human tumor cells. J Nat Prod, 2013; 76(8): 1413-23

16. Xiong WD, Gong J, Xing C: Ferruginol exhibits anticancer effects in OVCAR 3 human ovary cancer cells by inducing apoptosis, inhibition of cancer cell migration and G2/M phase cell cycle arrest. Mol Med Rep, 2017; 16(5): 7013-17

17. Jia Y, Wu C, Zhang B et al: Ferruginol induced apoptosis on SK-Mel-28 human malignant melanoma cells mediated through P-p38 and NF-אB. Human \& Experimental Toxicology, 2018

18. Da Rocha AB, Lopes RM, Schwartsmann G: Natural products in anticancer therapy. Curr Opin Pharmacol, 2001; 1(4): 364-69

19. Lopez J, Tait SW: Mitochondrial apoptosis: killing cancer using the enemy within. Br J Cancer, 2015; 112(6): 957-62 